\title{
Segmentação de coração em tomografias computadorizadas utilizando atlas probabilístico e redes neurais convolucionais
}

\author{
João O. B. Diniz ${ }^{1,2}$, Jonnison L. Ferreira ${ }^{1}$, Giovanni L. F. da Silva ${ }^{1,3}$, \\ Darlan B. P. Quintanilha ${ }^{1}$, Aristófanes C. Silva ${ }^{1}$, Anselmo C. Paiva ${ }^{1}$ \\ ${ }^{1}$ Núcleo de Computação Aplicada (NCA) \\ Universidade Federal do Maranhão (UFMA) \\ Caixa Postal 65.085-580 — São Luís — MA — Brasil \\ ${ }^{2}$ Instituto Federal de Educação, Ciência e Tecnologia do Maranhão (IFMA) \\ Caixa Postal 65.940-000 - Grajaú — MA — Brasil \\ ${ }^{3}$ Unidade de Ensino Superior Dom Bosco (UNDB) \\ Caixa Postal 65075-441 — São Luís - MA — Brasil \\ \{joao.bandeira, jonnison, giovannilucca, dquintanilha, ari, paiva\}@nca.ufma.br
}

\begin{abstract}
Organs at risk (OARs) are healthy tissues around cancer that must be preserved in radiotherapy $(R T)$. The heart is one of the fundamental OARs, so computer software has been developed to assist specialists in the segmentation. In this work, we proposed an automatic method for segmentation in computed tomography. The method consists of 3 steps: (1) acquisition of a public and diversified database; (2) volume standardization using histogram matching and rigid registration; and (3) segmentation of the heart using atlas and U-Net with residual blocks (ResU-Net). Thus, we achieved $92.53 \%$ of Dice and $84.73 \%$ of Jaccard. With the innovation and the results, it is shown that the proposed method is promising.
\end{abstract}

Resumo. Órgãos em risco (OARs) são tecidos saudáveis ao redor do câncer que devem ser preservados na radioterapia (RT). O coração é um dos OARs fundamentais, assim, softwares computacionais foram desenvolvidos para auxiliar os especialistas na segmentação. Neste trabalho, propõe-se um método automático para segmentação a partir da tomografia computadorizada. $O$ método consiste em 3 etapas: (1) aquisição de banco de dados público e diversificado; (2) padronização de volume usando registro e correspondência de histograma; e (3) segmentação do coração usando atlas e U-Net com blocos residuais (ResU-Net). Assim, alcançou-se 92,53\% de Dice e 84,73\% de Jaccard. Com a inovação e os resultados, mostra-se que o método proposto é promissor.

\section{Introdução}

O câncer ainda é uma das doenças mais letais. Câncer é o nome de mais de uma centena de doenças, que geralmente mostram distúrbios do crescimento celular que atacam tecidos e órgãos. Essas células se dividem rapidamente e geralmente são muito agressivas e incontroláveis, levando à formação de tumores e se espalhando para outras partes do corpo [St Germain et al. 2020]. 
A Radioterapia (RT) é um dos tratamento de câncer mais viáveis, sendo aplicada em aproximadamente 50\% dos casos de câncer [Evans and Staffurth 2018]. De acordo com [Gómez-Millán et al. 2015], a radioterapia pode ser a forma de tratar o câncer, com base na geometria do tumor e no nível do câncer. Essa solução é amplamente utilizada, especialmente quando a quimioterapia é muito arriscada para o paciente. Ainda assim, de acordo com [Evans and Staffurth 2018], 40\% dos pacientes curados do câncer tiveram RT como parte de seu tratamento.

O processo de RT consiste em algumas etapas, uma das etapas mais importantes sendo o planejamento. Os tratamentos contra o câncer são tóxicos, o que não é diferente da RT [Button and Staffurth 2010]. Em resumo, os raios ionizantes são enviados ao tecido-alvo para erradicar ou controlar o progresso do câncer. Porém, essa liberação de raios precisa ser bem planejada, visto que, ao redor desse tecido-alvo, existem tecidos/órgãos sensíveis à radiação. O excesso de radiação em órgãos saudáveis pode causar efeitos indesejáveis ou até irreversíveis [Evans and Staffurth 2018].

Para o planejamento, o paciente é submetido a uma tomografia, comumente chamada de tomografia de planejamento. Nessa tomografia, um grupo médico multidisciplinar, liderado por um dosimetrista, é responsável por identificar o tecido-alvo e os tecidos saudáveis que o circundam. Órgãos saudáveis são conhecidos como órgãos em risco (OARs) [Tsang and Hoskin 2017]. Esses OARs precisam ser segmentados pela equipe no planejamento da tomografia. O objetivo é criar um limite aceitável para a dose de raios no processo de $\mathrm{RT}$.

Dependendo da localização e do órgão alvo, uma série de outros OARs são delimitados de forma a não causar efeitos indesejados [Gómez-Millán et al. 2015]. Na segmentação desses órgãos, alguns softwares médicos são utilizados. O trabalho realizado por [La Macchia et al. 2012] comparou três softwares comerciais que fazem segmentação OAR. Eles mostram o uso dos softwares VelocityAI, MIM 5.1.1 e ABAS 2.0. O trabalho mostra que, apesar do uso de softwares, os melhores resultados só são alcançados com correção manual do especialista nos três softwares.

Assim, a busca por métodos computacionais para a segmentação automática de OARs ainda é um campo aberto de pesquisa. Entre os OARs, o coração mostra-se um dos órgãos mais importantes a serem protegidos, principalmente por seu papel essencial para o ser humano. A RT pode causar alterações no sistema cardiovascular, esse efeito é conhecido como cardiotoxicidade e pode se desenvolver em alguns dias, meses ou anos após a radiação. A cardiotoxicidade pode reduzir a qualidade de vida do paciente e aumentar o risco de morte por causas relacionadas ao coração [Piroth et al. 2019].

Assim, este trabalho propõe um método automático de segmentação do coração como OARs no planejamento tomográfico. O método proposto tem a função de servir de ferramenta de auxílio aos especialistas no processo de TR.

O método é dividido em três etapas. Primeiro, os TCs de planejamento a serem usados são adquiridos de um repositório público e diversificado. Em seguida, é padronizado em níveis de textura e tamanho dos volumes, uma vez que são adquiridos de diferentes maneiras. Posteriormente, a segmentação do coração é feita usando atlas e aprendizado profundo. 
Com os resultados alcançados pelo método de segmentação automática do coração, acredita-se que ele consegue algumas contribuições:

1. Uma técnica de pré-processamento para padronizar os volumes adquiridos de vários institutos diferentes;

2. Um método automático capaz de extrair uma região de interesse com base em atlas;

3. Uma segmentação do coração por uma técnica de aprendizado profundo usando blocos residuais e uma função de custo mista baseada em entropia cruzada e Dice.

Além desta seção, este artigo apresenta mais cinco seções. A próxima seção será a discussão dos trabalhos relacionados à tarefa de segmentação de coração destacando os avanços do método proposto em relação aos já publicados (Seção 2). A Seção 3 detalhou os materiais e o método para segmentação do coração. Os resultados e discussões são apresentados na Seção 4. A Seção 5 apresenta as conclusões e trabalhos futuros.

\section{Trabalhos Relacionados}

A segmentação automática de OARs ainda é um campo de pesquisa muito estudado, e vários métodos foram propostos ao longo do tempo. A seguir, apresenta-se alguns dos trabalhos mais recentes e considerados o estado da arte na tarefa de segmentação do coração.

O trabalho de [Trullo et al. 2017] propôs um método para segmentar OARs no planejamento de TC. O método proposto utiliza duas redes de aprendizagem profunda e campos aleatórios condicionais (CRF) para a segmentação de vários OARs. Os experimentos são conduzidos em 30 TCs planejados, e o melhor resultado alcançado para o coração é o coeficiente Dice de 91\%. [Dong et al. 2019] propuseram um método baseado em U-Net e Generative Adversarial Network (GAN) para a segmentação de vários OARs. O resultado é aplicado a um banco de dados de TC de 35 pacientes, e os melhores resultados de Dice para segmentação de coração foram $87 \%$.

O método proposto por [Vesal et al. 2019] apresenta técnicas de aprendizado profundo compostas por blocos residuais e camadas dilatadas. O resultado para a segmentação do coração foi de $94,1 \%$, no entanto, os autores usaram o aumento de dados no conjunto de dados de teste. [Chen et al. 2019] propuseram o uso de duas U-Net 3D para a segmentação de OARs. O método é aplicado em 60 pacientes e os melhores resultados para segmentação do coração foram 93,3\% de Dice.

O método proposto por [Feng et al. 2020] também propõe o uso de redes de aprendizagem profunda para a segmentação de OARs. O método é aplicado a um banco de dados público de 36 pacientes. Porém, no treinamento, os autores inseriram informações de um banco de dados privado para aumentar o número de amostras. Os resultados alcançam 91\% de dados médios. Em [Zhang et al. 2020], foi proposto um método baseado em aprendizado profundo para a segmentação de OARs. Os resultados foram aplicados a um banco de dados privado de 1240 pacientes com TC alcançadno $88,5 \%$ de Dice.

Como pode-se ver nos trabalhos, embora vários proponham a segmentação dos OARs, a segmentação do coração ainda é uma tarefa que requer melhorias. A maioria 
dos trabalhos aplica técnicas de aprendizado profundo sem tratamento prévio do banco de dados e/ou pós-processamento para reduzir falsos positivos.

Assim, o presente trabalho propõe a utilização de técnicas de processamento de imagens para o pré-processamento do conjunto de dados. Além disso, sugere-se uma maneira automática de usar o atlas para a segmentação de uma região inicial de interesse. Com base na eficiência do uso de aprendizado profundo demonstrada em todos os trabalhos de segmentação OAR, utilizou-se técnicas de aprendizado profundo para segmentação final do coração usando Blocos Residuais em U-Net (ResU-Net) com função de custo misto.

\section{Método proposto}

Para a segmentação do coração como órgão de risco na TC, propõe-se um método totalmente automático que utiliza técnicas de processamento de imagens e aprendizado de máquina. O método está dividido em 3 etapas: (1) aquisição do banco de dados, utilizouse uma base pública e diversificada; (2) padronização de volumes, aplicou-se técnicas de processamento de imagens para eliminar possíveis ruídos e deformidades causadas pelo processo de aquisição das imagens; (3) Segmentação do coração, primeiro segmentou-se o volume de interesse (VOI) usando uma técnica baseada em atlas, depois um modelo de aprendizado profundo para a segmentação final do coração. A Figura 1 ilustra as etapas do método proposto.

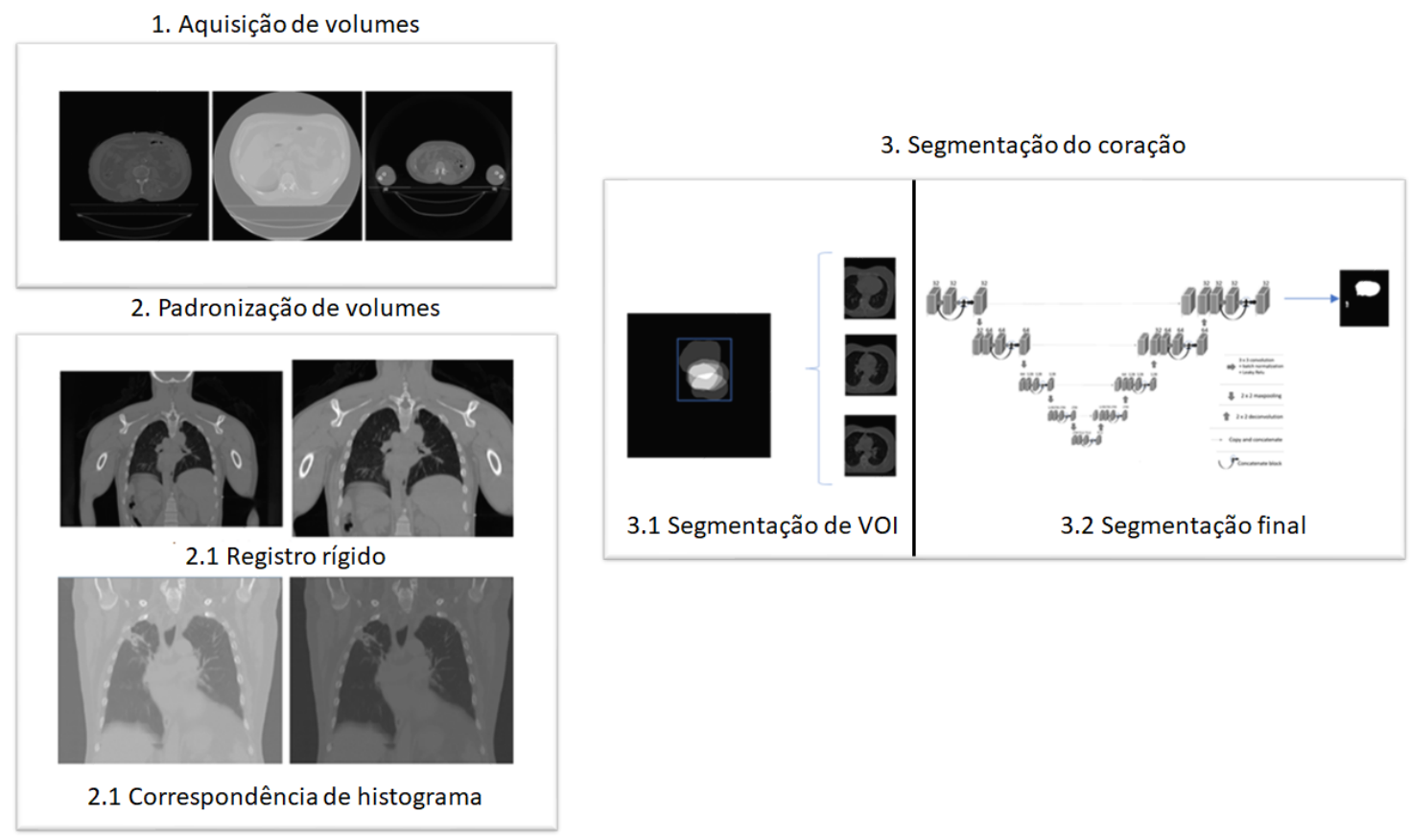

Figura 1. Fluxo de etapas do método

\subsection{Aquisição de volumes}

O banco de dados utilizado foi um público de TC com marcações de um especialista para o coração em 36 exames. Ele está disponível no The Cancer Imaging Archive, referenciado pelo nome LSTSC. As imagens são obtidas em três institutos diferentes com diversos 
protocolos, com número de fatias por exame variando de $134-288$. A escolha desta base de dados para a criação de um modelo de segmentação justifica-se pela diversidade. Figura 2 mostra um exemplo de fatia de cada instituto que compõe o banco de dados.
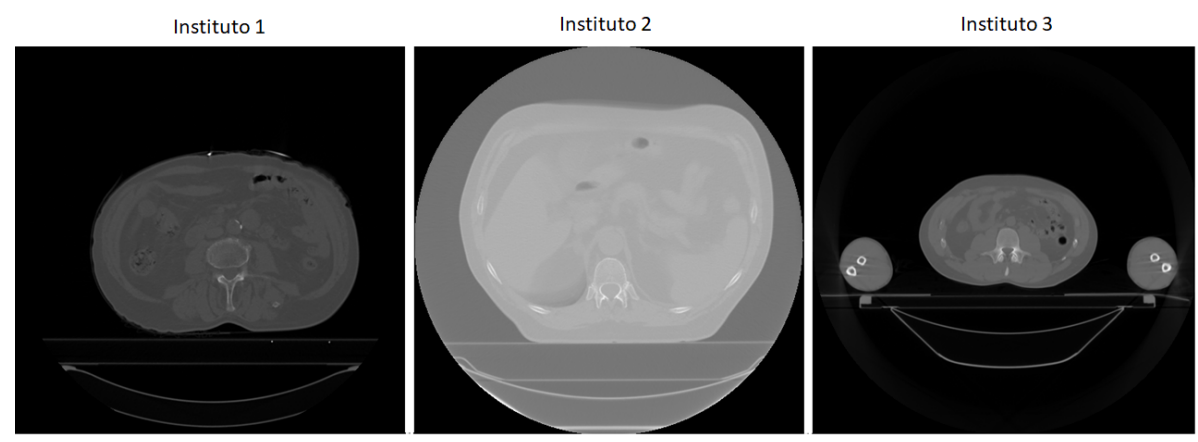

Figura 2. Aquisição de volumes

\subsection{Padronização de volumes}

Para resolver o problema do tamanho de cada paciente deixou-se todos os volumes do mesmo tamanho utilizando a técnica de registro rígido. Para tratar a diferença de voxels, utilizou-se a técnica de correspondência de histograma. A seguir, explica-se resumidamente cada uma dessas técnicas.

Uma forma de padronizar o número de fatias é a utilização do processo de registro. Neste trabalho, aplicou-se o registro rígido [Yoo 2004] a todo o banco de dados para que todas as imagens fossem movidas para o mesmo plano geométrico. Considerouse aquele com as menores fatias como um volume fixo. Este critério foi introduzido porque aproximar as fatias de volume com poucas fatias para uma com várias fatias produziria vários voxels que podem não estar corretos, uma vez que alguma interpolação seria necessária para aproximar esses volumes.

Observa-se, por ser composta de vários institutos e com vários exemplos de aquisições e uma variedade de voxels, há necessidade de normalizar os volumes, para que o método possa ser mais poderoso na percepção de padrões de textura de volume. Para isso, é proposta a estratégia de correspondência de histograma (ou especificação de histograma).

Em processamento de imagens, a especificação do histograma é a transformação de uma imagem de forma que seu histograma corresponda a um histograma especificado. A especificação do histograma pode ser usada para equilibrar as respostas do sistema de aquisição e normalizar duas imagens quando as imagens foram adquiridas por diferentes fontes de luz, sensores, clima ou iluminação [Gonzalez and Woods 2010]. Para isso, utilizou-se um volume correspondente para ser a base, um volume alvo que será transformado e, como resultado, tem-se um volume com o histograma correspondente.

O resultado da etapa de padronização de volume pode ser visto na Figura 3.

\subsection{Segmentação do coração}

Com volumes padronizados, o próximo passo é a segmentação do coração. Para isso, propõe-se a utilização de duas técnicas. A primeira define um volume de interesse usando 


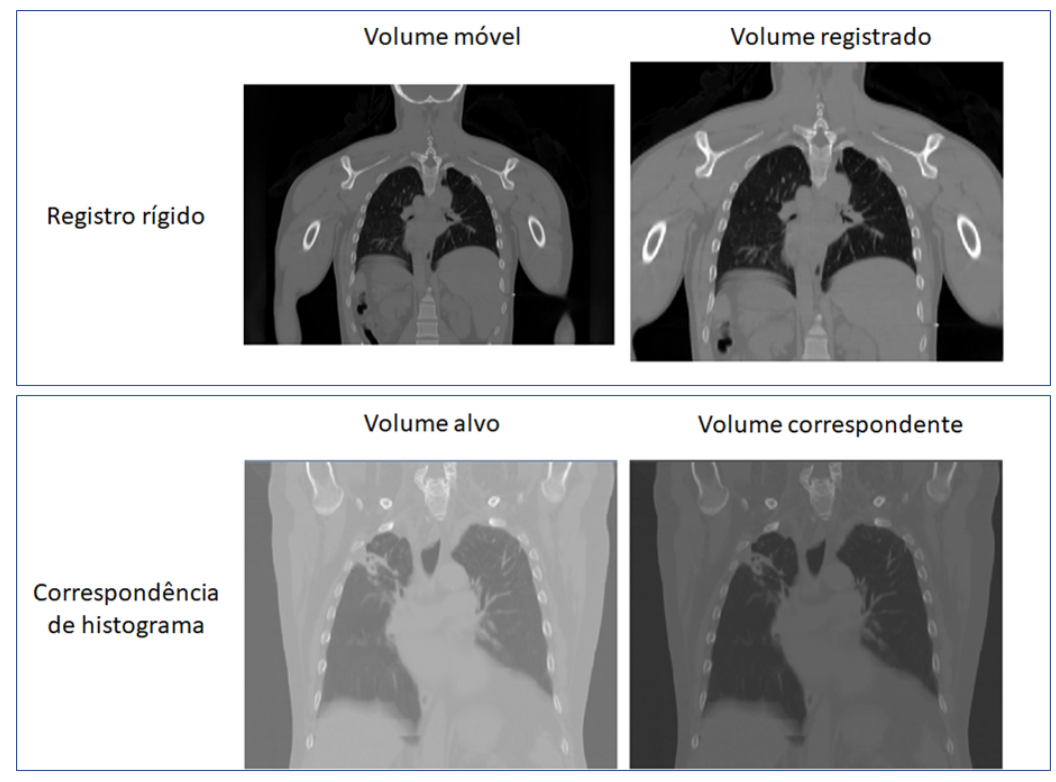

Figura 3. Etapa de padronização de volume

atlas. A segunda, faz-se a segmentação final do coração e utilizando uma rede neural convolucional com blocos residuais e uma função de perda mista composta de Dice e entropia cruzada binária.

\subsubsection{Segmentação de VOI}

Anatomicamente falando, o coração de cada paciente está localizado em uma região bem definida. Porém, dada a posição de cada paciente, altura, peso, entre outros fatores humanos, a localização deste OAR pode variar. Assim, propõe-se o uso de um atlas probabilístico para encontrar uma região provável de ser o coração. Para a confecção do atlas, foram utilizadas as marcações registradas dos pacientes. Assim, os valores do atlas são calculados a partir da média do valor do voxel nas marcações registradas. Com isso, tem-se um volume único com a probabilidade da existência do coração.

Com este volume criado, define-se a maior bounding box 3D que abrange todo o atlas. Então, todos os volumes do paciente são "cortados"para o tamanho correspondente a esta bounding box 3D. Desta forma, um VOI de interesse é segmentada com tamanho de volume reduzido.

Essa etapa é importante por dois motivos. Primeiro, utiliza-se de informações anatômicas e reais para diminuir a região de interesse, evitando previsões em regiões erradas. Em segundo lugar, sabe-se que durante o processo de utilização de redes de segmentação semântica, várias operações de subamostragem podem levar à perda de informações quando o objeto a ser detectado é pequeno em relação à imagem inteira. Isso ocorre no caso do coração, que corresponde a apenas uma pequena parte da TC completa do paciente.

O resultado da etapa de segmentação baseada em Atlas de VOI pode ser visto na Figura 4. 


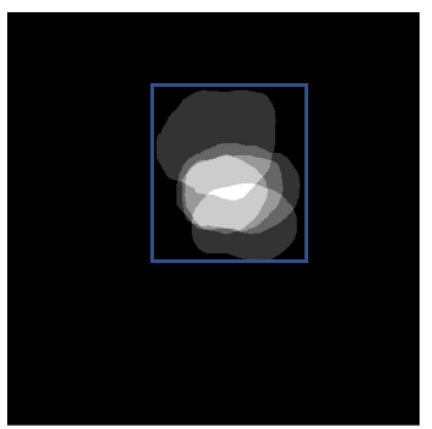

Bounding box do atlas
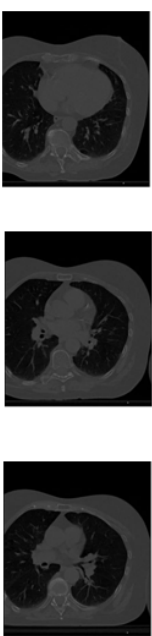

Volumes cortados

Figura 4. Segmentação de VOI. Com o Altas criado, é encontrado a maior bounding box que contém o objeto, em seguida, todos os exames são cortados neste mesm tamanho.

\subsubsection{Segmentação Final}

Para a segmentação do coração, utilizou-se uma abordagem bidimensional, ou seja, as fatias dos subvolumes serão as entradas para treinamento e classificação da rede, esta abordagem foi utilizada para aumentar o número de amostras e reduzir o poder computacional.. Assim, divide-se o banco de dados em três conjuntos: treinamento, validação e teste. Para isso, mantém-se o equilíbrio nos três conjuntos de dados garantindo que haja amostras dos três diferentes institutos em cada um dos conjuntos de dados.

No treinamento, propõe-se o uso de uma variante de rede de segmentação semântica U-Net. U-Net foi proposta para segmentação de órgãos em imagens médicas por [Ronneberger et al. 2015]. A U-Net, uma rede neural convolucional (CNN), usa os conceitos e camadas de uma CNN, isso inclui camadas convolucionais, pooling, ativação e dropout. CNNs estão sendo cada vez mais sendo usadas em vários problemas envolvendo imagens médicas [Paiva et al. 2019, Diniz et al. 2018, Diniz et al. 2019, da Cruz et al. 2020]. Neste trabalho, utilizou-se uma variação da U-Net, onde são inseridos blocos residuais a cada camada, chamada de ResU-Net. Apesar de existirem várias variações de U-Net com blocos residuais, neste trabalhos aplicou-se a proposta e explicada em [Diniz et al. 2020].

Além disso, observou-se que o uso de função de perda Dice não foi capaz de generalizar todos os exemplos da base de dados, sem gerar resultados significativos. Esta observação foi feita verificando os resultados do conjunto de dados de validação. Assim, aplicou-se uma combinação de entropia cruzada binária e a função de perda Dice [Sudre et al. 2017].

Com o modelo criado pela ResU-Net, o mesmo é aplicado a base de teste e as métricas de validação são calculadas. Neste trabalho utilizou-se as métricas Dice, Jaccard (JAC), Sensibilidade (SEN), Especificidade (ESP) e Acurácia (ACU). 


\section{Resultados e Discussões}

Nesta seção, descreve-se o resultado obtido com a aplicação do método proposto. Primeiro, apresenta-se o ambiente do treinamento. Em seguida, apresenta-se o resultado de cada etapa. Por fim, é apresentado o desempenho do método no estudo de caso e comparação com a literatura.

\subsection{Ambiente de trenamento}

Todo o método foi desenvolvido em Python. Para as etapas de padronização de volume, a biblioteca ITK foi usada. Na etapa de segmentação, utilizou-se funções NumPy para segmentação baseada em Atlas. Para a construção do ResU-Net, a biblioteca Keras com o back-end do TensorFlow em uma GPU equipada com RTX2070.

\subsection{Resultados das etapas do método}

O banco de dados, descrito na Seção 3.1, possui 36 volumes de três institutos distintos. Cada instituto com seu protocolo de aquisição. Assim, para obter exemplos de todos os institutos de treinamento, bem como de validação e teste, dividiu-se os conjuntos de dados para 9 pacientes de cada instituto no treino ( 27 volumes), 1 de cada na validação ( 3 volumes) e 2 de cada no teste (6 volumes).

$\mathrm{Na}$ etapa de padronização de volume (Seção 3.2) todos os pacientes passaram pelo registro e correspondência de histograma, logo, ao final dela tem-se os pacientes com mesmo tamanho e textura aproximada.

Com o atlas definido com marcações, todos os volumes foram cortados no tamanho da maior bounding box 3D que compreendia o atlas. Assim, todos os volumes foram cortados para um $224 \times 224 \times 60$, reduzindo as informações de treinamento e previsão em mais da metade.

Após várias seções de treinamento, e ajuste de parâmetros, os escolhidos na fase de treinamento foram: número de épocas igual a 100, tamanho de batch igual a 2, otimizador Adam com taxa de aprendizado inicial igual a 0,0001. Esses parâmetros foram os que apresentaram os melhores resultados no conjunto de dados de validação. Depois que o modelo é construído, ele é aplicado ao conjunto de dados de teste e os resultados são coletados.

Para validar o método proposto, foram feitos quatro cenários de simulações de treinamento: (1) sem a etapa de padronização e sem a segmentação de VOI; (2) apenas a padronização sem segmentação de VOI; (3) método completo substituindo a ResUNet pela U-Net de [Ronneberger et al. 2015]; e (4) método proposto. Os resultados são apresentados na Tabela 1.

Tabela 1. Resultados da segmentação do coração.

\begin{tabular}{cccccc}
\hline Cenário & Dice(\%) & JAC(\%) & SEN(\%) & ESP(\%) & ACU(\%) \\
\hline 1 & 56,35 & 44,22 & 58,22 & 96,33 & 91,03 \\
2 & 74,68 & 63,32 & 72,19 & 98,18 & 94,18 \\
3 & 87,85 & 78,34 & 87,59 & 99,09 & 98,67 \\
\hline 4 & 92,53 & 84,73 & 91,81 & 99,23 & 98,95 \\
\hline
\end{tabular}


Pode-se observar que a tarefa de padronização é essencial, produzindo uma melhora de quase $20 \%$ de valor de Dice do cenário 1 para o cenário 2. Isto demonstra a problemática existente nos protocolos de aquisição, uma vez minimizado este impacto por técnicas de processamento de imagens, os resultados tende a melhorar.

Após utilizar a padronização e a segmentação da VOI pelo atlas, foi realizado uma simulação usando a U-Net tradicional (cenário 3). Observou-se que estas duas etapas foram cruciais, apresentando resultados promissores de $87.85 \%$. Contudo, ao utilizar a ResU-Net (cenário 4), os resultados são ainda melhores. O conjunto de técnicas propostas para compor o método consegue alcançar resultados de Dice de 92.53\%. Vale destacar que prioriza-se a métrica Dice, pois esta que reflete o quanto a marcação do especialista é próxima a segmentação automática.

\subsection{Estudo de caso}

Nesta seção, apresenta-se o resultado de algumas fatias de um paciente com a marcação do especialista juntamente com a segmentação do método. A Figura 5 apresenta esse resultado.
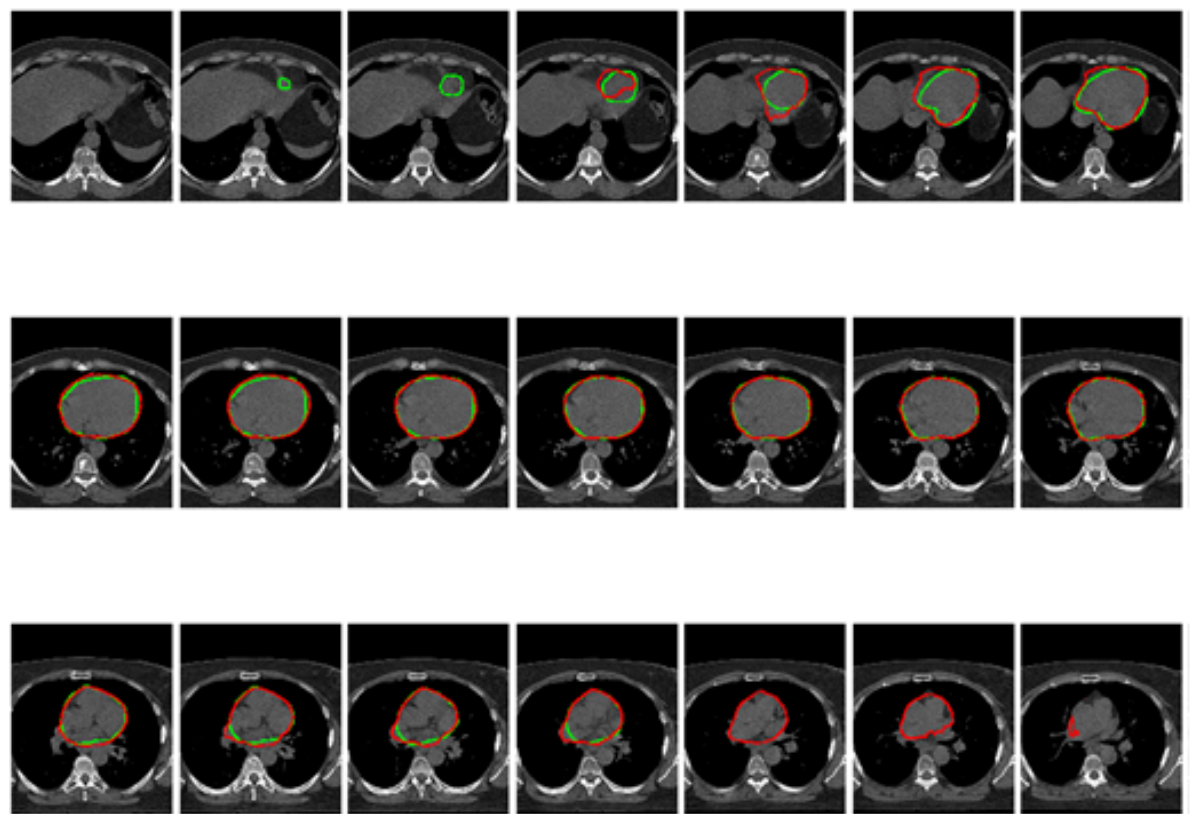

Figura 5. Estudo de caso: algumas fatias de um paciente da base de teste

Ao olhar para a Figura 5, destaca-se o quão eficaz é o método. A segmentação do método é muito próxima à marcação do especialista, em alguns casos apresentando uma sobreposição completa. No entanto, ainda existem regiões em que o especialista avaliou o coração, mas o método não conseguiu segmentar. O processo inverso também ocorre, em algumas regiões, o método considera coração pelas características próximas às marcações, mas o especialista não marcava. Isso se reflete no resultado médio dos Dice de $92,53 \%$. Contudo, vale destacar que o método com bastante precisão pode ser crucial para poupar o tempo do especialista, que pode emprega-lo em outras tarefas, como o próprio tratamento do câncer. 


\subsection{Comparação com os trabalhos relacionados}

Comparou-se os resultados alcançados pelo método com os resultados apresentados na Seção 2 e estão detalhados na Tabela 2.

Tabela 2. Comparação com os trabalhos relacionados.

\begin{tabular}{c|c|c|c|}
\hline \hline Métodos & Técnicas & Exames & Dice \\
\hline \hline [Trullo et al. 2017] & 2 CNN + CRF & 30 & $91 \%$ \\
\hline [Dong et al. 2019] & U-Net + GAN & 36 & $87 \%$ \\
\hline [Vesal et al. 2019] & U-Net Residual & 36 & $94,1 \%$ \\
\hline [Chen et al. 2019] & 3D U-Net & 60 & $93,3 \%$ \\
\hline [Feng et al. 2020] & 3D U-Net & 36 & $91 \%$ \\
\hline [Zhang et al. 2020] & U-Net & 1240 & $88,5 \%$ \\
\hline Método Proposto & ResU-Net & 36 & $92,5 \%$ \\
\hline \hline
\end{tabular}

Destaca-se que esta comparação é muito difícil, principalmente pelo fato de serem bases diferentes, métodos diferentes. Contudo, ao observar a métrica de Dice, percebe-se que o método tem local de destaque na literatura. Na sua grande maioria, os trabalhos relacionados utilizam apenas técnicas de aprendizado profundo para a segmentação do coração. No método proposto, tratou-se o problema ocasionado pela aquisição de imagens e de dimensionalidade através do atlas.

Apesar de [Chen et al. 2019] apresentar resultado superior ao método proposto, sua base de dados possuia mais pacientes e a abordagem proposta era $3 \mathrm{D}$, que muitas vezes é difícil ser executada devido a complexidade que o modelo exige. Por sua vez, [Vesal et al. 2019] alcança Dice de 94.1\%, porém este trabalho utilizou aumento de dados tanto no treino quanto no teste, o que pode influenciar negativamente os resultados.

Desta forma, acredita-se que o conjunto de técnicas apresentadas produzem resultados significantes na segmentação de coração, mesmo em uma base de dados bem diversificada. Assim, utilizar este método em grandes centros médicos, pode ser de fundamental importância na tarefa de segmentação de OARs.

\section{Conclusão}

Neste trabalho, foi proposta uma etapa de Padronização de Volume, que utiliza técnicas de processamento de imagens para reduzir os impactos causados pelos processos de aquisição de banco de dados. Validou-se isso usando uma Aquisição de Banco de Dados diversificada, composta por três institutos diferentes. Além disso, apresenta-se uma segmentação que utiliza duas técnicas consideradas de ponta em uma tarefa de segmentação: Atlas e Aprendizado Profundo. Vale ressaltar que o trabalho apresentou métricas de mais de $92 \%$ de Dice. De acordo com os trabalhos relacionados, este resultado é bastante promissor.

Porém, ainda existem trabalhos futuros que podem aprimorar o método. Como usar algoritmos genéticos ou evolutivos para encontrar os melhores parâmetros para ResU-Net. Além disso, deve-se notar que a abordagem proposta para o modelo é 2D, fazer essa técnica em 3D pode melhorar os resultados. Por fim, para validar o método, pode-se aplicá-lo em outras bases de dados. 


\section{Referências}

Button, M. and Staffurth, J. (2010). Clinical application of image-guided radiotherapy in bladder and prostate cancer. Clinical Oncology, 22(8):698-706.

Chen, P., Xu, C., Li, X., Ma, Y., and Sun, F. (2019). Two-stage Network for OAR segmentation.

da Cruz, L. B., Araújo, J. D. L., Ferreira, J. L., Diniz, J. O. B., Silva, A. C., de Almeida, J. D. S., de Paiva, A. C., and Gattass, M. (2020). Kidney segmentation from computed tomography images using deep neural network. Computers in Biology and Medicine, page 103906.

Diniz, J. O. B., Diniz, P. H. B., Valente, T. L. A., Silva, A. C., and Paiva, A. C. (2019). Spinal cord detection in planning ct for radiotherapy through adaptive template matching, imslic and convolutional neural networks. Computer methods and programs in biomedicine, 170:53-67.

Diniz, J. O. B., Ferreira, J. L., Diniz, P. H. B., Silva, A. C., and de Paiva, A. C. (2020). Esophagus segmentation from planning ct images using an atlas-based deep learning approach. Computer Methods and Programs in Biomedicine, page 105685.

Diniz, P. H. B., Valente, T. L. A., Diniz, J. O. B., Silva, A. C., Gattass, M., Ventura, N., Muniz, B. C., and Gasparetto, E. L. (2018). Detection of white matter lesion regions in mri using slic0 and convolutional neural network. Computer methods and programs in biomedicine, 167:49-63.

Dong, X., Lei, Y., Wang, T., Thomas, M., Tang, L., Curran, W. J., Liu, T., and Yang, X. (2019). Automatic multiorgan segmentation in thorax ct images using u-net-gan. Medical physics, 46(5):2157-2168.

Evans, E. and Staffurth, J. (2018). Principles of cancer treatment by radiotherapy. Surgery-Oxford International Edition, 36(3):111-116.

Feng, X., Bernard, M. E., Hunter, T., and Chen, Q. (2020). Improving accuracy and robustness of deep convolutional neural network based thoracic oar segmentation. Physics in Medicine \& Biology, 65(7):07NT01.

Gómez-Millán, J., Lara, M. F., Generoso, R. C., Perez-Rozos, A., Lupiáñez-Pérez, Y., and Carmona, J. A. M. (2015). Advances in the treatment of prostate cancer with radiotherapy. Critical reviews in oncology/hematology, 95(2):144-153.

Gonzalez, R. C. and Woods, R. E. (2010). Digital Image Processing. Pearson Prentice Hall.

La Macchia, M., Fellin, F., Amichetti, M., Cianchetti, M., Gianolini, S., Paola, V., Lomax, A. J., and Widesott, L. (2012). Systematic evaluation of three different commercial software solutions for automatic segmentation for adaptive therapy in head-and-neck, prostate and pleural cancer. Radiation Oncology, 7(1):160.

Paiva, A. M., Diniz, J. O., Silva, A., and Paiva, A. (2019). Segmentação de vértebras e diagnóstico de fraturas em imagens de ressonância magnética utilizando u-net $3 \mathrm{~d}$ e deep belief network. In Anais do XIX Simpósio Brasileiro de Computação Aplicada à Saúde, pages 106-117, Porto Alegre, RS, Brasil. SBC. 
Piroth, M. D., Baumann, R., Budach, W., Dunst, J., Feyer, P., Fietkau, R., Haase, W., Harms, W., Hehr, T., Krug, D., et al. (2019). Heart toxicity from breast cancer radiotherapy. Strahlentherapie und Onkologie, 195(1):1-12.

Ronneberger, O., Fischer, P., and Brox, T. (2015). U-net: Convolutional networks for biomedical image segmentation. In International Conference on Medical image computing and computer-assisted intervention, pages 234-241. Springer.

St Germain, D., Denicoff, A., Torres, A., Kelaghan, J., McCaskill-Stevens, W., Mishkin, G., O'Mara, A., and Minasian, L. M. (2020). Reporting of health-related quality of life endpoints in national cancer institute-supported cancer treatment trials.

Sudre, C. H., Li, W., Vercauteren, T., Ourselin, S., and Cardoso, M. J. (2017). Generalised dice overlap as a deep learning loss function for highly unbalanced segmentations. In Deep learning in medical image analysis and multimodal learning for clinical decision support, pages 240-248. Springer.

Trullo, R., Petitjean, C., Nie, D., Shen, D., and Ruan, S. (2017). Joint segmentation of multiple thoracic organs in ct images with two collaborative deep architectures. In Deep Learning in Medical Image Analysis and Multimodal Learning for Clinical Decision Support, pages 21-29. Springer.

Tsang, Y. M. and Hoskin, P. (2017). The impact of bladder preparation protocols on post treatment toxicity in radiotherapy for localised prostate cancer patients. Technical Innovations and Patient Support in Radiation Oncology, 3:37-40.

Vesal, S., Ravikumar, N., and Maier, A. (2019). A 2d dilated residual u-net for multiorgan segmentation in thoracic ct. arXiv preprint arXiv:1905.07710.

Yoo, T. S. (2004). Insight into images: principles and practice for segmentation, registration, and image analysis. AK Peters/CRC Press.

Zhang, J., Yang, Y., Shao, K., Bai, X., Fang, M., Shan, G., and Chen, M. (2020). A unetbased research on the multi-output convolution neural network's ability of decreasing mis-identification: Automatic segmentation of organs at risk in thorax. 